WŁodZIMIERz SzWARC (Milwaukee, WI)

\title{
ON CYCLING IN THE SIMPLEX METHOD OF THE TRANSPORTATION PROBLEM
}

Abstract. This paper shows that cycling of the simplex method for the $m \times n$ transportation problem where $k-1$ zero basic variables are leaving and reentering the basis does not occur once it does not occur in the $k \times k$ assignment problem. A method to disprove cycling for a particular $k$ is applied for $k=2,3,4,5$ and 6 .

1. Introduction. It is well known ([1], [2], [5], [6]) that cycling does occur in the simplex method of linear programming when this method generates a segment of non-optimal basic solutions $X, X_{1}, \ldots, X_{t}$ where $X=$ $X_{1}=\cdots=X_{t}$ and $X$ and $X_{t}$ share the same basis. Then the optimal solution can never be reached since this segment is replicated infinitely many times. To prevent cycling a special slightly modified linear programming problem is solved instead.

B. J. Gassner [4] presented two $4 \times 4$ and $5 \times 5$ assignment examples where cycling does occur. However, she did not apply the classical rule where the cell with the largest negative reduced cost enters the basis at each iteration.

G. B. Dantzig [3] in his 2003 book co-authored with M. N. Thapa posed the following open question while discussing Gassner's [4] result:

Does cycling occur for the simplex method in the transportation problem if the classical rule is applied to the original problem? In other words, does the simplex method of the transportation problem guarantee the optimality of the resulting solution? The paper addresses this particular issue.

We take advantage of the classical rule to establish the following result (Sections 3 and 4): To disprove cycling for the transportation problem, it is sufficient to show that it does not occur for a special $k \times k$ assignment

2000 Mathematics Subject Classification: 90C05, 90C08, 90C27.

Key words and phrases: transportation problem, assignment problem, cycling, basic solution, node, link, directed weighted tree. 
problem that deals with basic solutions $X=\left\{x_{i j}\right\}$ where

(a) the only positive elements $x_{i j}$ are on the main diagonal,

(b) the reduced cost is non-negative for at least one cell of each offdiagonal pair $(i, j),(j, i)$. The reduced costs of the basic cells are zero.

This result was established by using a surrogate simplex transportation method of [7] and [8] outlined in Section 2.

This paper presents a method of disproving cycling in a $k \times k$ assignment problem for a particular $k$. Consider all pairs $(i, j),(j, i)$ of off-diagonal cells. Mark a cell with a non-negative reduced cost by "+" and the other one by "-". The $k-1$ off-diagonal basic cells are marked by "+". Each basic solution $X$ is then described by a $k$-node weighted directed tree $T$. The trees turn out to be a convenient tool to generate all cycles for a given $k$. Cycling does not occur in the assignment problem if the trees form no cycle $T_{0}, T_{1}, \ldots, T_{t}$, $T_{0}=T_{t}$, or if for each cycle the respective solution sequence $X, X_{1}, X_{2}, \ldots$ terminates with an optimal basic solution $X_{u}, u<t$. The Appendix uses this method to disprove cycling for $k=2,3,4,5$ and 6 .

2. The surrogate simplex method for the transportation problem. The transportation problem deals with minimizing

$$
z(X)=\sum_{i=1}^{m} \sum_{j=1}^{n} c_{i j} x_{i j}
$$

subject to

$$
\begin{aligned}
& \sum_{j=1}^{n} x_{i j}=a_{i}, \quad i=1, \ldots, m, \\
& \sum_{i=1}^{m} x_{i j}=b_{j}, \quad j=1, \ldots, n, \\
& x_{i j} \geq 0,
\end{aligned}
$$

where $a_{i}>0, b_{j}>0$, and $c_{i j}$ are given numbers with $\sum_{i=1}^{m} a_{i}=\sum_{j=1}^{n} b_{j}$.

A loop $L$ is a set of $2 k$ cells $(i, j), 2 \leq k \leq \min (m, n)$, arranged in a sequence $\left(i_{1}, j_{1}\right),\left(i_{1}, j_{2}\right),\left(i_{2}, j_{2}\right), \ldots,\left(i_{k}, j_{k-1}\right),\left(i_{k}, j_{1}\right)$. Let $B$ be a set of $m+n-1$ cells $(i, j) . B$ is a basis if none of its subsets forms a loop. A matrix $X(B)=\left\{x_{i j}(B)\right\}$ is a basic feasible solution and $B$ a feasible basis if $X(B)$ satisfies $(2)-(4)$ and $x_{i j}(B)=0$ for each $(i, j) \notin B$. It is known that for each basis $B$ there exists a unique matrix $X$ that satisfies (2) and (3). The simplex method generates basic feasible solutions

$$
X(B), X\left(B_{1}\right), X\left(B_{2}\right), \ldots,
$$


leading to an optimal solution. Notice that replacing the $c_{i j}$ of (1) with $c_{i j}+u_{i}+v_{j}$ where the $u_{i}$ and $v_{j}$ are arbitrary numbers results in an equivalent transportation problem since $z(X)$ changes by a constant $\sum_{i=1}^{m} a_{i} u_{i}+$ $\sum_{j=1}^{n} b_{j} v_{j}$ for every $X$. The surrogate simplex method of [7] and [8] generates for each basis of (5) matrices $\left\{c_{i j}+u_{i}+v_{j}\right\}$ where $c_{i j}+u_{i}+v_{j}=0$ for each $(i, j) \in B$. Such matrices are symbolized by $C(B)=\left\{c_{i j}(B)\right\}$. Then the $-u_{i}$ and $-v_{j}$ are dual variables associated with $B$ and the original $c_{i j} . X(B)$ is optimal if all $c_{i j}(B) \geq 0$ since $z^{\prime}[X(B)]=\sum_{i} \sum_{j} c_{i j}(B) x_{i j}(B)=0 \leq z^{\prime}(X)$ for every $X$. Once $\min _{i, j \notin B} c_{i j}(B)=-c_{1}<0$ a new feasible solution $X\left(B_{1}\right)$ is generated. A matrix $C\left(B_{1}\right)$ is created by adding $c_{1}$ or $-c_{1}$ to certain rows and columns of $C(B)$. To illustrate this procedure consider Figure 1.

$C(B)=$\begin{tabular}{|c|c|c|c|}
\hline 0 & -2 & 0 & 4 \\
\hline 0 & 0 & -1 & 0 \\
\hline 2 & -3 & 0 & -5 \\
\hline
\end{tabular}

Fig. 1

Here $B=\{(1,1),(1,3),(2,1),(2,2),(2,4),(3,3)\}, \min c_{i j}(B)=-5$, and $B_{1}=B+(3,4)-(1,1)$. To get $C\left(B_{1}\right)$ add 5 to columns 1,2 and 4 , and -5 to row 2 of $C(B)$. This can also be accomplished by adding 5 to rows 1 and 3 , and -5 to column 3 of $C(B)$.

3. Problem reduction. Recall that cycling occurs in the transportation problem if the simplex method generates a sequence of feasible bases

$$
B, B_{1}, B_{2}, \ldots, B_{t}, B_{t+1}, \ldots,
$$

where $B=B_{t}$ and $\min _{(i, j) \notin B_{s-1}} c_{i j}\left(B_{s-1}\right)=-c_{s}<0$ for all $s \geq 1\left(B_{0}=B\right)$. Notice that $c_{s}$ is the maximum implicit cost of $C=\left\{c_{i j}\right\}$ for $B_{s-1}$ and $z\left[X\left(B_{r}\right)\right]$ is constant for each $X\left(B_{r}\right)$ of $(6)$.

A cell $(i, j) \in B$, where $x_{i j}(B)=0$, is called white if it leaves and reenters $B$, and black if it stays in every basis of (6).

THEOREM 1. Each cycling in an $m \times n$ transportation problem where $k-1$ cells reenter $B=B_{t}$ of (6) can be replicated in some $k \times k$ transportation problem where all $k$ diagonal cells stay in the initial basis $B^{\prime}$ while $k-1$ off-diagonal cells leave and reenter $B^{\prime}$.

Proof. Let $W$ and $B-W$ be sets of white and black cells respectively. $B-W$ is composed of $k$ subsets $V_{1}, \ldots, V_{k}$ where two black cells are assigned to the same $V_{s}$ if they share the same row or column. Notice that for each $(i, j) \in W, x_{u j}(B)>0$ and $x_{i v}(B)>0$ for some $u$ and $v$. Rearrange for convenience the rows and columns so that the set $V_{1}$ is situated in rows $I_{1}=$ 
$\left\{1, \ldots, m_{1}\right\}$ and columns $J_{1}=\left\{1, \ldots, n_{1}\right\}, V_{2}$ in rows $I_{2}=\left\{m_{1}+1, \ldots, m_{2}\right\}$ and columns $J_{2}=\left\{n_{1}+1, \ldots, n_{2}\right\}$, and so on.

Let $A_{11}, A_{22}, \ldots, A_{k k}$, where $A_{s s}=I_{s} \times J_{s}$, be areas occupied by $V_{1}, \ldots, V_{k}$ respectively. The white cells lie outside the $A_{s s}$ areas. Figure 2 presents an $8 \times 12$ matrix $C(B)$ where $W=\{(2,7),(1,9),(4,11)\}$ and $I_{1}=\{1,2,3\}$, $J_{1}=\{1,2,3\}, I_{2}=\{4,5\}, J_{2}=\{4,5,6,7\}, I_{3}=\{6,7\}, J_{3}=\{8,9,10\}$, $I_{4}=\{8\}, J_{4}=\{11,12\}$.

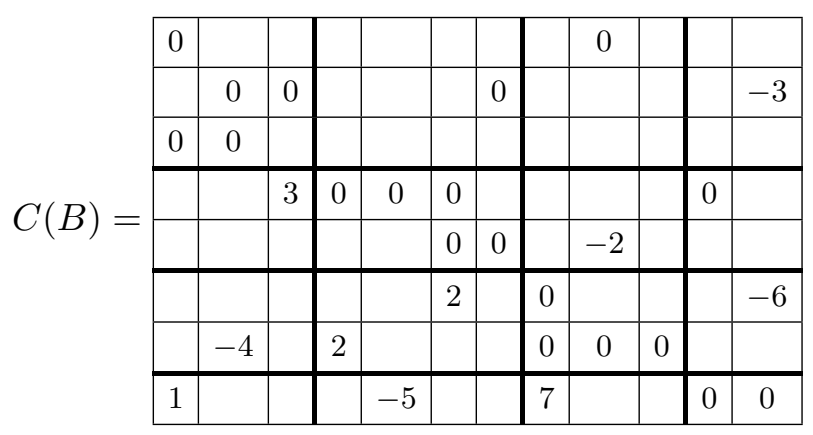

Fig. 2

Figure 2 presents only the basic entries $c_{i j}(B)=0$ and $\min c_{i j}(B)$ for each $A_{u v}=I_{u} \times J_{v}$ that does not contain the cells of $B$. A white cell links $V_{r}$ and $V_{s}$ if it shares a row with a black cell of $V_{r}$ and a column with a black cell of $V_{s}$. Notice that two cells of $W$ cannot link the same pair $V_{r}$ and $V_{s}$ since $B$ would contain a loop. Since the black cells stay in every basis of (6), $c_{i j}(B)=c_{i j}\left(B_{s}\right)=0$ for each $(i, j) \in A_{u u}$ and each $u$ and $k$. This can only happen if $C\left(B_{s}\right)$ is a result of adding to $c_{i j}(B):-d_{1}$ to each row of $I_{1}$ and $d_{1}$ to each column of $I_{1},-d_{2}$ to each row of $I_{2}$ and $d_{2}$ to each column of $J_{2}$, and so on. Then for each $(i, j) \in A_{u v}, c_{i j}\left(B_{s}\right)=c_{i j}(B)+d_{u}-d_{v}$. This implies that only the cells with $\min _{(i, j) \in A_{u v}} c_{i j}\left(B_{s}\right)$ can enter $B_{s+1}$ as white cells. This also includes the white cells which left $B$ and entered some basis of (6). Notice that for each $A_{r s}$ area with $r \neq s$ only cells with the smallest $c_{i j}(B)$ may leave and reenter $B$ when cycling occurs. We squeeze each $A_{r s}$ area into a single cell and define a $k \times k$ matrix $C^{\prime}\left(B^{\prime}\right)$ by placing $\min _{(i, j) \in A_{r s}} c_{i j}(B)$ in cells $(r, s)$ and 0 in cells $(r, r)$. Recall that for a white cell of $A_{r s}$ this minimum is zero. $B^{\prime}$ consists of $k$ black cells $(r, r)$ and $k-1$ white cells. Figure 3 presents $C^{\prime}\left(B^{\prime}\right)$ derived from the $8 \times 12$ matrix $C(B)$ of Figure 2 .

Here $B^{\prime}=\{(1,1),(2,2),(3,3),(4,4),(1,2),(1,3),(2,4)\}$.

Consider $B_{1}=B-\left(i_{1}, j_{1}\right)+\left(i_{2}, j_{2}\right)$ where $\left(i_{1}, j_{1}\right)$ links $V_{r}$ and $V_{s}$ and $\left(i_{2}, j_{2}\right)$ links $V_{p}$ and $V_{q}$. Since $-c_{1}=c_{i_{2} j_{2}}(B)=\min c_{i j}(B)=c_{p q}^{\prime}\left(B^{\prime}\right)$ cell $(p, q)$ enters $B_{1}^{\prime}$. Next we examine the loops $L$ and $L^{\prime}$ contained in $B+\left(i_{2}, j_{2}\right)$ and $B^{\prime}+(p, q)$ respectively. Let $\left(u_{1}, v_{1}\right), S,\left(u_{2}, v_{2}\right)$ be a segment of $L$ where $\left(u_{1}, v_{1}\right)$ and $\left(u_{2}, v_{2}\right)$ are white cells while $S$ is a sequence of black cells of $V_{h}$. 


$C^{\prime}\left(B^{\prime}\right)=$\begin{tabular}{|c|c|c|c|}
\hline 0 & 0 & 0 & -3 \\
\hline 3 & 0 & -2 & 0 \\
\hline-4 & 2 & 0 & -6 \\
\hline 1 & -5 & 7 & 0 \\
\hline
\end{tabular}

Fig. 3

The following four cases are possible: 1) $\left.u_{1}, u_{2} \in I_{h}, 2\right) v_{1}, v_{2} \in J_{h}$, 3) $\left.v_{1} \in J_{h}, u_{2} \in I_{h}, 4\right) u_{1} \in I_{h}, v_{2} \in J_{h}$. It is easy to see that the number of cells in $S$ is even for cases 1 and 2, and odd for cases 3 and 4. Suppose that $\left(u_{1}, v_{1}\right)$ and $\left(u_{2}, v_{2}\right)$ link: $V_{h}$ with $V_{k_{1}}$ and $V_{h}$ with $V_{k_{2}}$ (case 1$) ; V_{k_{1}}$ with $V_{h}$ and $V_{k_{2}}$ with $V_{h}$ (case 2); $V_{k_{1}}$ with $V_{h}$ and $V_{h}$ with $V_{k_{2}}$ (case 3 ); $V_{h}$ with $V_{k_{1}}$ and $V_{k_{2}}$ with $V_{h}$ (case 4). Then in $L^{\prime},\left(u_{1}, v_{1}\right), S,\left(u_{2}, v_{2}\right)$ corresponds to a segment: $\left(h, k_{1}\right),\left(h, k_{2}\right)$ (case 1$) ;\left(k_{1}, h\right),\left(k_{2}, h\right)$ (case 2$) ;\left(k_{1}, h\right),(h, h),\left(h, k_{2}\right)$ (case 3$)$ and $\left(h, k_{1}\right),(h, h),\left(k_{2}, h\right)$ (case 4$)$. Notice that $(h, h)$ is a black cell. Thus if the number of cells of $\left(u_{1}, v_{1}\right), S,\left(u_{2}, v_{2}\right)$ is even (odd) then it remains so in the corresponding segment of $L^{\prime}$. Since $\left(i_{1}, j_{1}\right)$ leaves $B$, there are an even number of cells in both $\left(i_{2}, j_{2}\right), \ldots,\left(i_{1}, j_{1}\right)$ segments of $L$. This number remains even for both $(p, q), \ldots,(r, s)$ segments of $L^{\prime}$. Thus cell $(r, s)$ that corresponds to $\left(i_{1}, j_{1}\right)$ can also be deleted from $B^{\prime}$.

The $c_{r s}^{\prime}\left(B_{1}^{\prime}\right)$ coincide with the $\min _{A_{r s}} c_{i j}\left(B_{1}\right), r \neq k$, for a simple reason. If $c_{1}$ is added to column $s$ of $C^{\prime}\left(B_{1}^{\prime}\right)$ then it is also added to each column of $J_{s}$ of the matrix $C\left(B_{1}\right)$. Then $-c_{1}$ is added to each row $s$ of $C^{\prime}\left(B_{1}^{\prime}\right)$ and each row of $I_{s}$ of $C\left(B_{1}\right)$. Thus cycling in sequence (6) can be replicated by the sequence $B^{\prime}, B_{1}^{\prime}, \ldots, B_{t}^{\prime}, \ldots$, where $B^{\prime}=B_{1}^{\prime}$.

To illustrate the second part of the proof, consider Figure 2, where $B_{1}=$ $B-(2,7)+(6,12) . B+(6,12)$ contains a 16-cell loop $L$ that passes the $A_{r s}$ areas in order $A_{34}, A_{33}, A_{13}, A_{11}, A_{12}, A_{22}, A_{24}, A_{44}$. In addition to $(6,12)$ it involves three white cells, four cells of $V_{1}$, three cells of $V_{2}$, three cells of $V_{3}$, and two cells of $V_{4}$. According to cases $1-4, L^{\prime}=(3,4),(3,3),(1,3),(1,2),(2,2)$, $(2,4)$ (see Figure 3$)$. Since $(2,7)$ links $V_{1}$ and $V_{2}$ and $(6,12)$ links $V_{2}$ and $V_{4}$, we have $B_{1}^{\prime}=B_{1}^{\prime}-(1,2)+(2,4)$. To get $C^{\prime}\left(B_{1}^{\prime}\right)$ we add 6 to columns 2 and 4 , and -6 to rows 2 and 4 of $C^{\prime}\left(B^{\prime}\right)$. The same numbers are added to each column of $J_{2}$ and $J_{4}$ and row $I_{2}$ and $I_{4}$ of $C(B)$ in order to get $C\left(B_{1}\right)$.

4. Properties of $k \times k$ matrices $C\left(B_{r}\right)$. From now on we assume that cycling occurs in a $k \times k$ transportation problem where each basis of (6) consists of $k$ black cells $(i, i), i=1, \ldots, k$, and $k-1$ white cells which leave $B$ and enter some subsequent bases. Consider the formula $c_{i j}\left(B_{r}\right)=$ $c_{i j}(B)+u_{i}+v_{j}$. Since all $c_{i i}\left(B_{r}\right)=0$ we have $u_{i}=-v_{i}$. Then

$$
c_{i j}\left(B_{r}\right)=c_{i j}(B)+v_{j}-v_{i} .
$$


Suppose a basic off-diagonal cell $(p, q)$ leaves $B_{r-1}$. Define a subset $A$ of $B_{r-1}-(p, q)$ as follows: 1$)$ each $(i, q)$ of the set $B_{r-1}-(p, q)$ belongs to $A$, $2)$ if $(i, j)$ belongs to $A$ then so does each cell $(u, j)$ and $(i, v)$. Let $J_{r-1}$ be the set of columns of $A$.

Notice that if $(i, j)$ replaces $(p, q)$ in $B_{r}$ then both $q$ and $j$ belong to $J_{r-1}$, while $i$ and $p$ do not. To get $C\left(B_{r}\right)$ we add $c_{r}$ and $-c_{r}$ to the columns and rows of $J_{r-1}$ of $C\left(B_{r-1}\right)$.

Notice that the $v_{j}$ satisfy the condition

$$
v_{j}=\sum_{s \in \alpha_{j}} c_{s}
$$

where $\alpha_{j}$ is a subset of $\{1, \ldots, r\}$.

THEOREM 2. Suppose that for some $r$ all white cells left $B$ (some may have reentered later). Then for each $(p, q)$ with $p \neq q$ and some $s \geq r$ either $c_{p q}\left(B_{r}\right)=c_{p q}\left(B_{s-1}\right)+c_{s}$ or $c_{q p}\left(B_{r}\right)=c_{q p}\left(B_{s-1}\right)+c_{s}$.

Proof. Suppose $(p, q)$, the basic cell of $B_{r}$, entered $B_{s}$ and stayed basic in $B_{s+1}, B_{s+2}, \ldots, B_{r}$. Then $c_{p q}\left(B_{s-1}\right)=-c_{s}$ and $c_{p q}\left(B_{r}\right)=c_{p q}\left(B_{s}\right)=$ $c_{p q}\left(B_{s-1}\right)+c_{s}$. Next assume that $(p, q) \notin B_{r}$. Then $B_{r}+(p, q)$ contains a loop $L=(p, q),\left(p, j_{2}\right),\left(i_{2}, j_{2}\right), \ldots,\left(i_{u}, j_{u-1}\right),\left(i_{u}, q\right)$. Assign all odd cells of $L$ to $L_{1}$ and all even cells to $L_{2}$. Then for any $u_{i}$ and $v_{j}$,

$$
\sum_{L_{1}} c_{i j}-\sum_{L_{2}} c_{i j}=\sum_{L_{1}}\left(c_{i j}+u_{i}+v_{j}\right)-\sum_{L_{2}}\left(c_{i j}+u_{i}+v_{j}\right) .
$$

Notice that $c_{i j}\left(B_{r}\right)=0$ for each $(i, j) \in L-(p, q)$. Hence $c_{p q}\left(B_{r}\right)=$ $\sum_{L_{1}} c_{i j}\left(B_{r}\right)-\sum_{L_{2}} c_{i j}\left(B_{r}\right)$.

Suppose all cells of $L-(p, q)$ belong to $B_{s}, B_{s+1}, \ldots, B_{r}$ and $(k, l)$ is one of its cells that entered $B_{s}$. Then $c_{k l}\left(B_{s-1}\right)=-c_{s}<0$ and $c_{i j}\left(B_{s-1}\right)=0$ for each $(i, j) \in L-(p, q)-(k, l)$. Due to $(9)$,

$$
c_{p q}\left(B_{r}\right)=\sum_{L_{1}} c_{i j}\left(B_{s-1}\right)-\sum_{L_{2}} c_{i j}\left(B_{s-1}\right) .
$$

Then

$$
c_{p q}\left(B_{r}\right)= \begin{cases}c_{p q}\left(B_{s-1}\right)+c_{s} & \text { if }(k, l) \in L_{2}, \\ c_{p q}\left(B_{s-1}\right)-c_{s} & \text { if }(k, l) \in L_{1} .\end{cases}
$$

If $c_{p q}\left(B_{r}\right)=c_{p q}\left(B_{s-1}\right)-c_{r}$, then $c_{q p}\left(B_{r}\right)=c_{q p}\left(B_{s-1}\right)+c_{r}$.

We call $(p, q)$ a "+" (resp. "-") cell if it satisfies the first (resp. second) condition of (10). There are $\frac{1}{2} k(k-1)$ cells in each category. Notice that $c_{p q}\left(B_{r}\right) \geq 0$ for each "+" cell. Only "-" cells can replace the "+" cells in a basis.

COROllary 1. Under the assumption of Theorem $2, \alpha_{p} \neq \alpha_{q}$ for each $p \neq q, p, q=1, \ldots, k$. 
Proof. Suppose $\alpha_{p}=\alpha_{q}$. Then $u_{p}+v_{q}=-v_{q}+v_{q}=0$ and $c_{p q}\left(B_{r}\right)=$ $c_{p q}\left(B_{u}\right)$ for each $u \leq r$, contrary to the fact that $(p, q)$ is either a "+" or "-" cell.

Since cycling occurs we can expand sequence (6) to $B_{-t}, B_{-t+1}, \ldots$, $B_{-1}, B, B_{1}, \ldots, B_{t}, \ldots$, where the initial basis is $B_{-t}=B=B_{t}$. This is why we can assume that Theorem 2 holds for $C\left(B_{u}\right)$ of any $B_{u}$ of (6).

We say that column $j$ dominates column $i$ in $C\left(B_{u}\right)$ if $v_{j}-v_{i}=c_{r}+L\left(c_{r}\right)$ for some $r \leq u$, where $L\left(c_{r}\right)=a_{1} c_{1}+a_{2} c_{2}+\cdots+a_{r-1} c_{r-1}$ and $a_{i}$ are 0,1 and -1 .

Due to Corollary 1, for each $C\left(B_{u}\right)$ either column $j$ dominates $i$ or $i$ dominates $j$. Notice that $(7)$ implies that $(i, j)$ of $C\left(B_{u}\right)$ is a " + " cell if column $j$ dominates column $i$. Otherwise $(i, j)$ is a "-" cell. Then for each $C\left(B_{u}\right)$ one can list the columns such that the number of " + " cells is $0,1,2, \ldots, k-1$ respectively. Use the following rule. Assign weight $w_{i}=r$ to column $i$ if there are $r-1$ "+" cells in this column. Then $(i, j)$ is a "+" cell of $C\left(B_{u}\right)$ if $w_{i}<w_{j}$ and a "-" cell if $w_{i}>w_{j}$. We can assume that all "+" cells of $C(B)=C\left(B_{t}\right)$ are above the main diagonal since this can be done by rearranging the rows and columns of $C(B)$. Then $w_{i}=i$ for each column $i$. Notice that all $(i, j)$ of $C(B)$ below the main diagonal are "-" cells.

Consider a basis $B^{*}$ where each white basic cell appears in a separate row and column. We prove the following:

Property 1. $B^{*}$ does not belong to sequence (6).

Proof. Suppose $B^{*}=B_{u}$ for some $B_{u}$ of (6). We can rearrange the rows and columns of $C\left(B^{*}\right)$ so that all cells above (resp. below) the main diagonal are "+" (resp. "-") cells. Let $(i, j)$ satisfy $c_{i j}\left(B_{u}\right)=-c_{u+1}$. Then $B_{u+1}=B_{u}+(i, j)-(p, q)$ for some cell $(p, q)$. Then (see proof of Theorem 2), $B_{u}+(i, j)$ contains a loop $L=L_{1}+L_{2}$ where $(i, j)$ belongs to the set $L_{1}$ of odd elements of $L$ and $(p, q)$ belongs to $L_{2}$, the set of even elements of $L$. Since all elements of $L_{2}$ are black diagonal cells, $z\left(B_{u+1}\right)=z\left(B_{u}\right)-c_{u+1}$. Hence $B^{*}$ cannot appear in (6) where $z\left(B_{u}\right)$ is constant for each $u=0,1, \ldots, t$, where $B_{0}=B$.

5. Directed weighted trees. Since the columns of the matrices $C\left(B_{u}\right)$ have assigned distinct weights we are in a position to define a corresponding $k$-node weighted directed tree $T_{u}$ as follows:

Each column $i$ of $C\left(B_{u}\right)$ is a node of $T_{u}$ while each white basic cell $(i, j)$ is a directed link $i \rightarrow j$ of $T_{u}$ where $w_{i}<w_{j}$ since $(i, j)$ is a "+" cell of $C\left(B_{u}\right)$. Link $i \rightarrow j$ will also be described as $\left[w_{i}\right] i \rightarrow\left[w_{j}\right] j$. Now, instead of $C(B), C\left(B_{1}\right), \ldots, C\left(B_{t}\right)$ where $C(B)=C\left(B_{t}\right)$ we deal with a sequence, 
called cycle:

$$
T_{0}, T_{1}, \ldots, T_{t}, \quad \text { where } T_{0}=T_{t},
$$

where each link of $T_{0}$ leaves and reenters $T_{0}$.

For convenience assume that $w_{i}=i$ in $T_{0}$. Then all cells $(i, j), i<j$, of $C(B)$ are "+" cells. Let $T^{*}$ be the tree corresponding to $C\left(B^{*}\right)$ of Property 1 . Then $T^{*}=i_{1} \rightarrow i_{2} \rightarrow i_{3} \rightarrow \cdots \rightarrow i_{k-1} \rightarrow i_{k}$ where the weights of nodes $i_{1}, \ldots, i_{k}$ are $1, \ldots, k$. Due to Property $1, T^{*}$ is of the type $(1,2),(2,3), \ldots,(k-1, k)$ and cannot appear in cycle $(11)$. Next consider any tree $T$ other than $T^{*}$. To construct a successor $T^{\prime}$ of $T$ apply the following procedure:

1. Remove a link $p \rightarrow q$ from $T$ thus splitting it into two non-empty subtrees.

2. Let $A$ and $\bar{A}$ be sets of nodes of the two subtrees.

3. Assign $p$ to $A$ and $q$ to $\bar{A}$. Assume $|A|=m$. Obviously $m \geq 1$.

4. Select nodes $i$ of $A$ and $j$ of $\bar{A}$ where $w_{i}>w_{j}$.

5. Define $T^{\prime}$ as follows: Replace $p \rightarrow q$ with $i \rightarrow j$. Distribute new weights $w_{i}^{\prime}$ in $T^{\prime}$ by assigning weights $1,2, \ldots, m$ to the nodes of $A$ and $m+1$, $\ldots, k$ to the nodes of $\bar{A}$ so that for each pair $(r, s)$ belonging to the same subset $A$ or $\bar{A}, w_{r}^{\prime}<w_{s}^{\prime}$ if $w_{r}<w_{s}$.

Due to $4, T^{*}$ has no successors.

Let $T_{0}$ correspond to $C(B)$. Then $(p, q)$ is a " + " basic white cell while $(i, j)$ is a "-" cell of $C(B)$. To disprove cycling consider each of the $k^{k-2}$ trees $T_{0}$ where $w_{i}=i$. For each $T_{0}$ we construct all possible sequences $T_{0}, T_{1}, T_{2}, \ldots$ where $T_{u+1}$ is a successor of $T_{u}$.

Definition. Trees $T_{s}$ and $T_{r}$ of (11) are equivalent if to every link $\left[w_{i}\right] i \rightarrow\left[w_{j}\right] j$ of $T_{s}$ there corresponds a link $\left[w_{i}\right] f(i) \rightarrow\left[w_{j}\right] f(j)$ of $T_{r}$ where $f(1), \ldots, f(k)$ is a permutation of numbers $1, \ldots, k$. The set of pairs $\left(w_{i}, w_{j}\right)$ describes their identical type.

Next we focus on those $T_{0}$ for which there exists a sequence

$$
T_{0}, T_{1}, T_{2}, \ldots, T_{r}
$$

where $T_{r}$ is the earliest tree equivalent to $T_{0}$.

Notice that $T_{u}$ and $T_{r+u}, u \geq 1$, are equivalent if for each $u$ the weights of nodes $i, j, p$, and $q$ in $T_{u}$ and $T_{r+u}$ are identical. Then (11) can also be written as

$$
T_{0}, \ldots, T_{r}, \ldots, T_{2 r}, \ldots, T_{h r}=T_{t}=T_{0},
$$

where $n \geq 1$ and $T_{0}, T_{r}, T_{2 r}, \ldots$, are equivalent trees.

Each of the $h$ sequences $T_{0}, \ldots, T_{r} ; T_{r}, \ldots, T_{2 r} ; \ldots ; T_{(h-1) r}, \ldots, T_{h r}$ is called a subcycle. 
To create a subcycle consider the tree

$$
T_{0}=[1] 1 \rightarrow[3] 3,[2] 2 \rightarrow[3] 3,[2] 2 \rightarrow[5] 5, \quad[4] 4 \rightarrow[5] 5,
$$

which in terms of $\left(w_{i}, w_{j}\right)$ is of the type $(1,3),(2,3),(2,5),(4,5)$. Remove from $T_{0}$ the link with weights 2 , 3, i.e. link $2 \rightarrow 3$. Then $A=\{1,3\}$ and $\bar{A}=\{2,4,5\}$. Select nodes with weights 4 and 3 , i.e. nodes 4 and 3 , and replace it with link $4 \rightarrow 3$ to create a successor tree $T_{1}=[1] 2 \rightarrow[3] 5,[2] 4 \rightarrow$ $[3] 5,[2] 4 \rightarrow[5] 3,[4] 1 \rightarrow[5] 3$.

Note that the tree $T_{0}$ has seven other successors.

$T_{1}$ is equivalent to $T_{0}$ since its $\left(w_{i}, w_{j}\right)$ type is also $(1,3),(2,3),(2,5),(4,5)$ where $f(1), f(2), f(3), f(4), f(5)=2,4,5,1,3$.

The shape of $T_{0}$ and $T_{1}$ can be represented as follows:

$$
[1] \rightarrow[3] \leftarrow[2] \rightarrow[5] \leftarrow[4],
$$

where the numbers indicate the weights of the nodes. Repeating the same procedure for $T_{1}$, where $p, q=4,5$ and $i, j=1,5, A=\{2,5\}$ and $\bar{A}=$ $\{1,3,4\}$ we get

$$
T_{2}=[1] 4 \rightarrow[3] 3, \quad[2] 1 \rightarrow[3] 3, \quad[2] 1 \rightarrow[5] 5, \quad[4] 2 \rightarrow[5] 5 .
$$

Continuing in the same fashion we get a cycle $T_{0}, T_{1}, \ldots, T_{6}$ where $T_{0}=T_{6}$.

Notice that cycling does not occur if no subcycle exists for any $T_{0}$. To study the other properties of subcycles it is sufficient to consider subcycle (12). Let $i \rightarrow j$ symbolize the link that enters $T_{u+1}$ by replacing some link of $T_{u}$. Link $i \rightarrow j$ varies with $u$. Let $w_{r, u-1}$ and $w_{r u}$ be the weights of node $r$ in $T_{u-1}$ and $T_{u}$. The following properties eliminate certain $T_{0}$ trees.

Property 2. Link $1 \rightarrow k$ does not appear in $T_{0}=T_{t}$.

Proof. Suppose $1 \rightarrow k$ belongs to $T_{0}$. Then $w_{01}=w_{l t}=1$ and $w_{k 1}=$ $w_{k t}=k$ and for some $u$, link $1 \rightarrow k$ reenters $T_{u+1}$ by replacing some link $p \rightarrow q$ of $T_{u}$ and stays in all subsequent trees $T_{u+1}, \ldots, T_{t}$. Suppose $w_{l, u+1}=1$ and $w_{k, u+1}=k$. Then $w_{p u}>w_{l u}>w_{k u}>w_{q u}$, which contradicts $w_{p u}$ $<w_{q u}$. This contradiction also holds when $p=1$ or $q=k$. Hence $w_{k, u+1}-$ $w_{l, u+1}<k-1$. But $w_{k, u+1}-w_{l, u+1} \geq w_{k, u+2}-w_{l, u+2} \geq \cdots \geq w_{k t}-w_{l t}=$ $k-1$, which contradicts the last inequality.

Property 3. Links $1 \rightarrow(k-1)$ and $2 \rightarrow k$ cannot simultaneously belong to $T_{0}$.

Proof. It is sufficient to show that each of those links can only reenter $T_{t}=T_{0}$.

Notice that during the transition from $T_{t-1}$ to $T_{t}$ we have $A=\{1, \ldots, m\}$ and $\bar{A}=\{m+1, m+2, \ldots, k\}$ for some $1 \leq m<k$. Suppose $1 \rightarrow(k-1)$ is in $T_{t-1}$. Then $A=\{1, \ldots, k-1\}, \bar{A}=\{k\}$ and $1<w_{k, t-1}<k$. Thus $w_{l, t-1}=1$ and $w_{k-l, t-1}=k$, contradicting Property 2 . If $2 \rightarrow k$ is in $T_{t-1}$ 
then $A=\{1\}, \bar{A}=\{2, \ldots, k\}$ and $1<w_{l, t-1}<k$. Then $w_{2, t-1}=1$ and $w_{k, t-1}=k$, again contradicting Property 2 .

Consider the sequence $C(B), C\left(B_{1}\right), \ldots, C\left(B_{u}\right)$ where $-c_{u+1}=c_{r s}\left(B_{u}\right)$ $=\min c_{i j}\left(B_{u}\right)$. Let $A_{m}$ and $\bar{A}_{m}$ be sets used in the transition from the tree $T_{m-1}$ to $T_{m}$. Then

$$
-c_{u+1}=c_{r s}(B)+a_{1} c_{1}+a_{2} c_{2}+\cdots+a_{u} c_{u},
$$

where

$$
a_{m}= \begin{cases}0 & \text { if } r \text { and } s \text { both belong to either } A_{m} \text { or } \bar{A}_{m}, \\ -1 & \text { if } r \text { belongs to } A_{m} \\ 1 & \text { if } r \text { belongs to } \bar{A}_{m}\end{cases}
$$

Notice that if $-c_{u+1}=c_{r s}\left(B_{u-1}\right)<0$ then $c_{u} \geq c_{u+1}$.

If $-c_{u+1}=c_{r s}\left(B_{u-1}\right)-c_{u}$, then $c_{u} \geq c_{u+1}$ if $c_{r s}\left(B_{u-1}\right) \geq 0$, and $c_{u}<c_{u+1}$ if $c_{r s}\left(B_{u-1}\right)<0$.

To disprove cycling for a particular $k$, consider the set of $k^{k-2}$ trees $T_{0}$ where $w_{i}=i$. From this set eliminate first those $T_{0}$ that satisfy Properties 2 and 3. For each remaining tree create a list of possible successors that are still on the list. Next eliminate trees whose only successor is $T^{*}$ and update the list of successors for the remaining trees.

Repeating this elimination process, we end up with a list of trees $T_{0}$ which participate in subcycle (12) which is a segment of cycle (11). If no $T_{0}$ remains on the list then each tree sequence $T_{0}, T_{1}, \ldots$, terminates with $T^{*}$ which corresponds to $X\left(B^{*}\right)$. Then, due to Property 1 , cycling does not occur in the assignment problem. Otherwise generate all possible cycles for each $T_{0}$.

Consider $-c_{u+1}$ defined by (13). We state the following.

Conjecture. Cycling does not occur in the assignment problem if for each tree cycle (11) there exists a $u<t$ such that $-c_{u+1} \geq 0$.

If for each cycle $(11),-c_{u+1}=\min c_{i j}\left(B_{u}\right) \geq 0$ for some $u<t$, then sequence (5) terminates with $X\left(B_{u}\right)$ which is an optimal basic solution. Hence cycling does not occur.

We prove in the Appendix that the Conjecture holds for $k=2,3,4,5$, and 6 .

6. Concluding remarks. This paper demonstrates that to disprove cycling for the transportation problem it is sufficient to disprove it for a very special $k \times k$ assignment problem with an arbitrary $k$. It also makes a Conjecture (Section 5) which guarantees that cycling does not occur. It presents a method of disproving cycling for a particular $k$ and shows that the Conjecture holds for $k \leq 6$. A challenging question remains how to prove 
this conjecture for an arbitrary $k$. So far only a few necessary conditions have been established that reduce the types of trees in a subcycle. The sufficient conditions might help to identify additional criteria when $-c_{u+1} \geq 0$ for $u<t$ in a cycle. According to the Appendix all 21 cycles for $k=5$ were handled by Criterion I while all but two out of 7083 cycles for $k=6$ were handled by Criteria I and II.

One should mention another property of trees. Let sym $x=k+1-x$ where $x$ is an integer $1, \ldots, k$. Define sym $T$ as a symmetrical tree of $T$ by replacing every link $\left[w_{i}\right] i \rightarrow\left[w_{j}\right] j$ of $T$ by the link $\left[\operatorname{sym} w_{i}\right] \operatorname{sym} i \leftarrow\left[\operatorname{sym} w_{j}\right] \operatorname{sym} j$. Notice that $w_{i}<w_{j}$ implies that $\operatorname{sym} w_{i}>\operatorname{sym} w_{j}$.

The following property holds: $T_{0}, T_{1}, \ldots, T_{r}$ is a subcycle if and only if $\operatorname{sym} T_{0}, \operatorname{sym} T_{1}, \ldots, \operatorname{sym} T_{r}$ is a subcycle. This result reduces considerably the number of $T_{0}$ types to identify subcycles.

Appendix: Proof that no cycling occurs for $k=2,3,4,5$ and 6 . To disprove cycling for a particular $k$, we identify the types of $T_{0}$ for which there exists a subcycle $(12)$. Hence type $(1,2),(2,3), \ldots,(k-1, k)$ that has no successors is not considered. We assume that $w_{i}=i$ in $T_{0}$.

CASE $k=2$. The set of types is empty.

CASE $k=3$. Due to Properties 2 and 3 , the set is empty.

CASE $k=4$. Properties 2 and 3 reduce the set of 16 types of $T_{0}$ to the following four types: $(1,2),(1,3),(3,4) ;(1,2),(2,3),(2,4) ;(1,2),(2,4),(3,4)$; and $(1,3),(2,3),(3,4)$. Each of the four types converges to the type $(1,2)$, $(2,3),(3,4)$ after at most two iterations. Thus no subcycle exists for $k=4$.

CASE $k=5$. We identify 14 types of $T_{0}$ for which there exists at least one subcycle (12). Recall that $w_{i}=i$ in $T_{0}$. The types form four groups: 1) 24,2$) 74,3) 14,38,42,100$, and 4) $21,25,39,45,49,72,73,99$. Here is the $\left(w_{i}, w_{j}\right)$ description of the 14 types.

$\begin{array}{rlll}24: & (1,2),(1,4),(3,4),(3,5) & 25: & (1,2),(1,4),(3,4),(4,5) \\ 74: & (1,3),(2,3),(2,5),(4,5) & 39: & (1,2),(2,3),(2,5),(4,5) \\ 14: & (1,2),(1,3),(3,4),(4,5) & 45: & (1,2),(2,4),(3,4),(3,5) \\ 38: & (1,2),(2,3),(3,4),(2,5) & 49: & (1,2),(2,5),(3,4),(4,5) \\ 42: & (1,2),(2,3),(3,5),(4,5) & 72: & (1,3),(2,3),(2,4),(4,5) \\ 100: & (1,4),(2,3),(3,4),(4,5) & 73: & (1,3),(2,3),(3,4),(2,5) \\ 21: & (1,2),(1,4),(2,3),(4,5) & 99: & (1,4),(2,3),(3,4),(3,5)\end{array}$

Tables 1-4 present $w_{i}, w_{j}, w_{p}$, and $w_{q}$ during the transition from $T_{u}$ to $T_{u+1}$ for each of the four groups. The upper entry is $w_{i}-w_{j}$ (in type 24 it is $3-2$ ), the lower entry is $w_{q}-w_{p}$ (i.e. $3-4$ in 24 ). 
Table 1 Table 2

\begin{tabular}{ccc}
24 & \multicolumn{2}{c}{74} \\
24 & 34 & $4-3$ \\
$3-4$ & 74 & $2-3$ \\
\hline
\end{tabular}

Table 1 means that if $T_{u}$ is of type 24 and link of weights $3 \rightarrow 2$ replaces link of weights $3 \rightarrow 4$, then $T_{u+1}$ is also of type 24 . The same goes for type 74 . If $T_{0}$ is of type 24 then $T_{0}=2 \leftarrow 1 \rightarrow 4 \leftarrow 3 \rightarrow 5$ where $w_{i}=i$. Since $3 \rightarrow 2$ replaces $3 \rightarrow 4$, we have $A=\{3,5\}$ and $\bar{A}=\{1,2,4\}$. Hence the weights in $T_{1}$ are 1,2 for nodes 3,5 of $A$ and $3,4,5$ for nodes $1,2,4$ of $\bar{A}$.

Tables 3 and 4 handle the other two types:

Table 3

\begin{tabular}{|c|c|c|c|}
\hline 14 & 38 & 42 & 100 \\
\hline 14 & $\begin{array}{l}3-2 \\
1-2\end{array}$ & & \\
\hline 38 & & $\begin{array}{l}5-4 \\
2-3\end{array}$ & \\
\hline 42 & & & $\begin{array}{l}4-3 \\
4-5\end{array}$ \\
\hline \begin{tabular}{l|l|}
\cline { 2 - 2 } 100 & $2-1$ \\
\end{tabular} & & & \\
\hline
\end{tabular}

Table 4

\begin{tabular}{|c|c|c|c|c|c|c|c|}
\hline 21 & 25 & 39 & 45 & 49 & 72 & 73 & 99 \\
\hline 21 & & $\begin{array}{l}4-3 \\
1-2\end{array}$ & & & & & \\
\hline 25 & & & & & $\begin{array}{l}3-2 \\
3-4 \\
\end{array}$ & $\begin{array}{l}3-2 \\
1-2 \\
\end{array}$ & \\
\hline 39 & & & $\begin{array}{l}4-3 \\
2-3\end{array}$ & & & & $\begin{array}{l}4-3 \\
4-5\end{array}$ \\
\hline 45 & $\begin{array}{l}3-2 \\
3-4\end{array}$ & & & $\begin{array}{l}5-4 \\
3-4\end{array}$ & & & \\
\hline 49 & $\begin{array}{l}3-2 \\
4-5\end{array}$ & & & & & & \\
\hline $\begin{array}{l}2-1 \\
2-3\end{array}$ & & $\begin{array}{l}4-3 \\
2-3 \\
\end{array}$ & & & & & \\
\hline 73 & & & & $\begin{array}{l}5-4 \\
2-3\end{array}$ & $\begin{array}{l}5-4 \\
3-4\end{array}$ & & \\
\hline $\begin{array}{l}2-1 \\
3-4\end{array}$ & & & $\begin{array}{l}2-1 \\
2-3\end{array}$ & & & & \\
\hline
\end{tabular}

Based on Tables 1, 2, 3 and 4 we prove the following:

PROPERTY 4. If cycling occurs for $k=5$ then $c_{u} \geq c_{u+1}$ for each $u$.

Proof. Consider a cycle (11) where $c_{u}<c_{u+1}$ for some $u$. Let $w_{i, u-1}$, $w_{j, u-1}$ and $w_{i u}, w_{j u}$ be the weights of nodes $i$ and $j$ in the trees $T_{u-1}$ and $T_{u}$ of (11). By assumption $(i, j)$ is a " $-"$ cell both in $C\left(B_{u-1}\right)$ and $C\left(B_{u}\right)$. 
Hence both differences $w_{i, u-1}-w_{j, u-1}$ and $w_{i u}-w_{j u}$ are positive. According to Tables $1-4$, for each $s, w_{i s}-w_{j s}=1$ when link $i \rightarrow j$ enters $T_{s+1}$. Since $c_{u}<c_{u+1}, i$ and $j$ belong to different sets $A$ and $\bar{A}, 0<w_{i, u-1}-w_{j, u-1}<$ $w_{i u}-w_{j u}=1$, which is impossible. Hence $c_{u} \geq c_{u+1}$.

Using Tables 1-4 we generate the following 21 subcycles:
1. 24,24
12. $21,39,99,45,49,25,72,21$
2. 74,74
13. $25,73,72,39,45,25$
3. $14,38,42,100,14$
14. $25,73,72,39,45,49,25$
4. $21,39,45,25,73,72,21$
15. $25,73,72,39,99,45,25$
5. $21,39,45,25,72,21$
16. $25,73,72,39,99,45,49,25$
6. $21,39,45,49,25,73,72,21$
17. $25,73,49,25$
7. $21,39,45,49,25,72,21$
18. $25,72,39,45,25$
8. $21,39,99,21$
19. $25,72,39,45,49,25$
9. $21,39,99,45,25,73,72,21$
20. $25,72,39,99,45,25$
10. $21,39,99,45,25,72,21$
21. $25,72,39,99,45,49,25$

11. $21,39,99,45,49,25,73,72,21$

The number of subcycles in a cycle varies from 2 to 4 . The largest 33-tree cycle is composed of four subcycles 11 (see the list above). The smallest 7-tree cycle is composed of seven identical type trees (of types 24, 74). To disprove cycling we have to show that for each tree cycle (11) there exists a $u<t$ where $-c_{u}=\min c_{i j}\left(B_{u-1}\right) \geq 0$. This means that $X\left(B_{u-1}\right)$ is an optimal solution.

Consider the cycle $T_{0}, T_{1}, \ldots, T_{6}$ where each tree is of type 74 , handled in Section 5. Using formula (13) we get $-c_{1}=c_{43}(B)<0, A_{1}=\{1,3\}$ and $-c_{2}=c_{15}(B)-c_{1}, A_{2}=\{2,5\}$. Consider $T_{2}$. To create $T_{3}$ replace link $p, q$ with weights 2,3 by a link with weights 4,3 ; i.e. $i, j=2,3,-c_{3}=$ $c_{23}(B)+c_{1}-c_{2} \geq 0$ since $(2,3)$ is a "+" cell of $C(B)$ and $c_{1} \geq c_{2}$ (Property 4 ).

Cycling is disproved for this tree cycle since $X\left(B_{2}\right)$ is an optimal solution.

Next consider the first four trees $T_{0}, T_{1}, T_{2}, T_{3}$ of the largest 33-tree cycle composed of subcycles 11. Here $-c_{1}=c_{43}(B), A_{1}=\{2,3\},-c_{2}=c_{25}(B)-c_{1}$, $A_{2}=\{1,3,4,5\},-c_{3}=c_{12}(B)+c_{1}-c_{2} \geq 0$. Again $X\left(B_{2}\right)$ is an optimal solution for this cycle tree.

For nine subcycles $-c_{4}=c_{15}(B)+c_{2}-c_{3} \geq 0$ while for the remaining eleven subcycles $-c_{3}=c_{i j}(B)+c_{1}-c_{2} \geq 0$ where $(i, j)=(1,2),(2,3)$ or $(3,4)$.

CASE $k=6$. There are 420 types of $T_{0}$ participating in at least one of the 7083 subcycles. The computer program used two criteria to identify cycles (composed of subcycles) where $-c_{u+1} \geq 0$ for some $u<t$. 
CRITERIOn I: There exist numbers $u$ and $r$ with $r<u-1$ such that

$$
-c_{u+1}=c_{i j}\left(B_{r}\right)+c_{u-1}-c_{u}, \quad c_{i j}\left(B_{r}\right) \geq 0 \quad \text { and } \quad c_{u-1} \geq c_{u} .
$$

Hence $-c_{u+1} \geq 0$.

CRITERION II: There exist numbers $u, r$ and $s$ with $s+1<r<u$ such that

$$
-c_{u+1}=c_{i j}\left(B_{r}\right)+c_{s}+c_{u-1}-c_{u}, \quad c_{i j}\left(B_{r}\right) \geq 0,
$$

where

$$
c_{s} \geq c_{s+1} \geq \cdots \geq c_{u-1} .
$$

To prove that $-c_{u+1} \geq 0$ it is sufficient to show that $d=c_{s}+c_{u-1} \geq 0$. Notice that $d \geq 0$ if $c_{u-1} \geq c_{u}$.

Consider the case $c_{u-1}<c_{u}$. Suppose cell $(p, q)$ enters the basis $B_{u}$. The assumption $c_{u-1}<c_{u}$ implies that $-c_{u}=c_{p q}\left(B_{u-1}\right)=c_{p q}\left(B_{u-2}\right)-c_{u-1}$ $\left(c_{p q}\left(B_{u-1}\right)=c_{p q}\left(B_{u-2}\right)\right.$ implies that $\left.c_{u-1} \geq c_{u}\right)$. Thus

$$
c_{u-1}-c_{u}=c_{p q}\left(B_{u-2}\right) \geq \min c_{i j}\left(B_{u-2}\right)=-c_{u-1} \geq-c_{s} .
$$

Thus $d \geq 0$. The computer program disproved cycling for 6894 subcycles by Criterion I and for 183 subcycles by Criterion II.

For the remaining two subcycles one or both of the conditions (16) and $c_{u-1} \geq c_{u}$ may not hold. There $-c_{8}=c_{56}(B)+c_{2}+c_{6}-c_{7}$ and $-c_{8}=$ $c_{12}(B)+c_{2}+c_{6}-c_{7}$. Here $c_{56}(B) \geq 0$ and $c_{12}(B) \geq 0$; however, the inequality $c_{2} \geq c_{3} \geq \cdots \geq c_{6} \geq c_{7}$ does not hold. The respective $-c_{7}$ for those subcycles happen to be identical: $-c_{7}=c_{25}(B)-c_{1}-c_{6}=c_{25}\left(B_{1}\right)-c_{6}$. Due to $c_{25}\left(B_{1}\right) \geq-c_{2}$ we get $-c_{7} \geq-c_{2}-c_{6}$. Hence $d \geq 0$ for both subcycles. This concludes the proof of Case $k=6$.

\section{References}

[1] E. M. L. Beale, Cycling in the dual simplex method, Naval Res. Logistics Quart. 2 (1955), 269-275.

[2] W. H. Cunningham and J. E. Klincewicz, On cycling in the network simplex method, Math. Programming 26 (1983), 182-189.

[3] G. B. Dantzig and M. N. Thapa, Linear Programming 2: Theory and Extensions, Springer Ser. Operations Res., 2003, p. 216.

[4] B. J. Gassner, Cycling in the transportation problem, Naval Res. Logistics Quart. 11 (1964), 43-58.

[5] A. J. Hoffman, Cycling in the simplex algorithm, National Bureau of Standards Report No. 2974, December 1953.

[6] K. T. Marshall and J. W. Suurballe, A note on cycling in the simplex method, Naval Res. Logistics Quart. 16 (1969), 121-137.

[7] W. Szwarc, Transportation problem, Zastos. Mat. 7 (1962), 149-187 (in Polish). 
[8] W. Szwarc, The solution of the transportation problem, Mathematica (Cluj) 4 (27) (1962), 149-157.

School of Business Administration

University of Wisconsin-Milwaukee

Milwaukee, WI 53201, U.S.A.

E-mail: wlodek.1@comcast.net

Received on 18.10.200\%;

revised version on 14.3.2008

(1895) 
